\title{
NOVO MÉTODO PARA CALCULAR O DIÂMETRO MÉDIO DE AGREGADOS DE SOLOS(1)
}

\author{
Q. de J ONG van LIER ${ }^{(2)} \&$ J . A. ALBUQUERQUE ${ }^{(3)}$
}

\begin{abstract}
RESUMO
Ao calcular o diâmetro médio ponderado (DMP) ou o diâmetro médio geométrico (DMG) de uma amostra de solo, ponderam-se o diâmetro médio aritmético (no caso do DMP) ou o logarítmico (no caso do DMG) de cada classe pela massa de agregados nela retida. A média aritmética de cada classe, multi plicada pela massa de agregados retidos na respectiva classe, apenas é correta, quando ocorrer uma distribuição si métrica de massa de agregados dentro de cada classe, o que é muito pouco provável. O objetivo deste trabalho, desenvolvido no final de 1996 no Departamento de Solos da UFRGS, Porto Alegre, é propor um novo método para o cálculo do diâmetro médio dos agregados, presumindo ser a distribuição dentro de cada classe semel hante à distri buição dos agregados de toda a amostra. E ssa proposta pressupõe ser o diâmetro médio da classe mais bem estimado por aquele diâmetro que divide os agregados contidos nela em duas partes de massa i gual. Para isso foi apresentado um procedi mento de cálculo, e o diâmetro médio assim encontrado foi denomi nado "diâmetro médio ajustado" (DMA). Para testar o método, utilizaram-se dados existentes de vários solos, condições de manejo do solo e rotações de culturas. Considerando os resultados, justifica-se a utilização do parâmetro DMA no lugar do DMP ou DMG, por representar mais corretamente o diâmetro médio dos agregados de uma amostra de solo.
\end{abstract}

Termos de indexação: diâmetro médio ponderado, diâmetro médio geométrico, diâmetro médio ajustado, estabilidade de agregados.

\section{SUMMARY: A NEW METHOD FOR THE CALCULATION OF THE MEAN DIAMETER OF SOIL AGGREGATES}

When cal culating themean weight-diameter (MWD) or thegeometric mean diameter (GMD) of a soil sample, the arithmetic mean diameter (in the case of MWD) or the logarithmic mean (in the case of GMD) of cach class is weighed by the mass of aggregates retained in that dass. The use of thearithmetic mean of each class, multiplied by theaggregatemass retained in that class is correct only if a symmetric distribution of aggregate sizes occurs within each class, which is very unlikely. I n this paper, devel oped by theend of 1996 at theSoil ScienceDepartment

\footnotetext{
${ }^{(1)}$ Trabalho apresentado na I Reunião Sul-Brasileira de Ciência do Solo, em Lages (SC), 9-10 de outubro de 1996. Recebido para publicação em março de 1997 e aprovado em outubro de 1997.

(2) Professor Adjunto do Departamento de Solos, UFRGS. Caixa Postal 776, CEP-90001-970 Porto Alegre (RS). Bolsista do CNPq. E-mail qdjvlier@vortex.ufrgs.br

${ }^{(3)}$ Estudante de Doutorado em Ciência do Solo, PPGAg/UFRGS. Professor Assistente, UDESC-CAV, Caixa Postal 28, CEP 88520-000 Lages (SC). E-mail a2ja@cav.udesc.br.
} 


\begin{abstract}
of the UFRGS, Porto Alegre, Brazil, a new method is proposed aiming the calculation of the mean diameter of aggregates of soil samples, assuming a si milar distributi on of aggregatesizes within each class and within the whole sample It is assumed that the mean diameter of each class is better estimated by that diameter that divides theaggregates contained in thecl ass in two parts with equal mass. A calculation procedure is presented and the calculated mean diameter is referred to as "mean adjusted diameter" (MAD). To evaluate the method, it is applied on existing data from different soils, management conditi ons and cropping rotations. It is concluded that theMAD should beused as a substitute of theMWD and GMD, as it represents the mean aggregate diameter of a soil sample in a more correct way.
\end{abstract}

Index terms: mean weight-diameter, geometric mean diameter, mean adjusted diameter, aggregatestability.

\section{INTRODUÇÃO}

A estabilidade dos agregados da camada superficial éum parâmetrofísico cuja importância é reconhecida por aquel es que estudam o processo de erosão do solo. Essa estabilidadedepende, principal mente, da textura do solo, da sua mineralogia, do teor etipos dematéria orgânica e da sua umidade. A determinação da distribuição dos tamanhos de agregados estáveis em água, técnica baseada no método deYoder (1936), com modificações recomendadas pelo Committee on Physical Analyses (Van Bavel, 1953), é descrita detal hadamente em Kemper \& Chepil (1965). Os vários métodos, com diferenças quanto ao tipo e quantidade deenergia fornecida ao sistema, utilizam, em geral, jogo de peneiras com diferentes aberturas de mal ha, nas quais se distribuem os agregados e as partículas.

Para medir e calcular os dados de análise de agregados, Van Bavel (1949) propôs construir uma curva de freqüência acumulada da massa de agregados de cada classe em função do limite superior da classe. A área acima da curva, que pode ser medida com planímetro, expressaria o diâmetro médio ponderado pela massa, atribuindo maior peso aos agregados de maior tamanho, considerados indicativos de boa estrutura para a maioria dos propósitos agronômicos.

O método de Van Bavel (1949) é trabalhoso e subjetivo, como relataram Schaller \& Stockinger (1953), comparando cinco métodos para expressar dados de agregação. Esses autores se mostraram favoráveis aos métodos que utilizam determinadas classes de diâmetro, como, por exemplo, agregados maiores que $2 \mathrm{~mm}$. Na prática, a fração maior que $2 \mathrm{~mm}$ podeser utilizada como um índice de agregação, por apresentar coeficiente de correl ação de 0,96 com o diâmetro médio ponderado (DMP, mm). No entanto, a determinação deve ser repetida diversas vezes por causa do alto coeficiente de variação. Assim, o uso de apenas uma peneira não traz economia de tempo no laboratório. Quando se utiliza um jogo de peneiras, a proposta mais simples para o cálculo do diâmetro médio é o DMP, calculado da seguinte maneira (Van Bavel, 1949):

$$
D M P=\sum_{i=1}^{n} \frac{m_{i}}{m} \cdot \bar{D}_{i}
$$

em que $\mathrm{n}$ é o número de classes de tamanho de agregados, $m_{i}$ éa massa da classe i, méa massa total e $\bar{D}_{i}$ é a média aritmética dos limites inferior e superior da classe.

A mel hor aproximação do ver dadei roval or do DM , segundo alguns autores, pode ser obtida por meio do diâmetro médio geométrico (DMG, $\mathrm{mm}$ ):

$$
D M G=\prod_{i=1}^{n} D_{i}^{\frac{m_{i}}{m}}=e^{\sum_{i=1}^{n} \frac{m_{i}}{m} \cdot \ln \left(\bar{D}_{i}\right)}
$$

Gardner (1956), avaliando a distribuição do tamanho de agregados em mais de 200 locais, observou, na maioria dos casos, acúmulo deagregados nas classes maiores. Em solos de regiões de clima tropical, a estabilidade dos agregados mostra-se, geralmente, superior à dos agregados de solos de regiões de clima temperado, resultando em distribuição assimétrica. Nesses casos, conforme o autor, o uso de papel log-probabilístico érecomendado. Plota-se o diâmetro inferior da dasse $(\mathrm{mm})$ em função da distribuição acumulada (g). Os resultados obtidos são expressos em termos de diâmetro médio geométrico (DMG) elogaritmo do desvio-padrão. Stirk (1958) questiona o uso de uma média, quando a distribuição dos agregados for diferente da normal (ou seja, assimétrica), eindica ser o uso do diâmetro médio geométrico mais apropriado. No método analítico, apresentado por Youker \& McGuinness (1957), foi proposto usar a média aritmética dos extremos de cada classe em vez do limite extremo da classe.

Até o presente momento, o DMG e o DMP são amplamente utilizados (Lal et al., 1980; Eltz et al., 1989; Carpenedo \& Mielniczuk, 1990; Unger, 1991; Ellsworth et al., 1991; Campos et al., 1995, Amezketa et al., 1996). O uso da média aritmética de cada classe multiplicada pela massa de agregados retidos na respectiva classe apenas é correta, quando se verificar uma distribuição simétrica de massa de agregados dentro de cada classe, o que é muito pouco provável.

O objetivo destetrabal hoé propor um novo método para o cálculo da distribuição dos agregados, presumindo ser a distribuição dentro de cada classe semelhante à distribuição dos agregados de toda a amostra. 


\section{MÉ TODO}

Ao contrário dos métodos usuais, em que se presume que o diâmetro médio de cada classe equivale à média aritmética entre os limites superior einferior dela, esta proposta parte do pressuposto que o diâmetro médio da classe é mais bem estimado pelo diâmetro que divide os agregados nela contidos em duas partes de massas iguais. Para determinar esses diâmetros médios das classes, tomaram-se, como base, as massas retidas em cada peneira. Procurou-se obter uma equação invertível que se ajustasse aos pares de dados do diâmetro superior da classe ( $D, \mathrm{~mm})$, bem como a fração da massa da classe acumulada com a das classes inferiores $\left(F, \mathrm{~g} \mathrm{~kg}^{-1}\right)$. Nestetrabal ho, utilizaram-se as seguintes equações:

$$
\begin{gathered}
F=\frac{1}{a+b / D} \\
F=\frac{1}{a+b / D^{0,5}} \\
F=a \cdot D^{b}
\end{gathered}
$$

em que $a$ e b são os parâmetros empíricos das respectivas equações. Com base nos resultados desta regressão, cal cularam-se os diâmetros médios de cada classe, invertendo-se a correspondente equação (3), (4) ou (5) e substituindo $F$ por $\left(F_{i}+F_{i+1}\right) / 2$, resultando, respectivamente, nas equações (6), (7) e (8):

$$
D_{i}=\frac{b \cdot\left(F_{i}+F_{i+1}\right)}{2-a \cdot\left(F_{i}+F_{i+1}\right)}
$$

$$
\begin{gathered}
D_{i}=\left[\frac{b \cdot\left(F_{i}+F_{i+1}\right)}{2-a \cdot\left(F_{i}+F_{i+1}\right)}\right]^{2} \\
D_{i}=\left[\frac{F_{i}+F_{i+1}}{2 a}\right]^{1 / b}
\end{gathered}
$$

em que $D_{i}$ é o diâmetro médio ajustado da classe $i, e$ $\mathrm{F}_{\mathrm{i}}$ e $\mathrm{F}_{\mathrm{i}+1}$ são as frações de massa acumulada das dasses i e i +1 , respectivamente.

Esse procedimento é ilustrado na figura 1, que representa os pares de dados obtidos por Carpenedo (1985), trabal hando com material de um latossol o roxo distrófico sob campo nativo com tratamento úmido. As linhas pontilhadas referem-se ao cál culo do diâmetro médio aritmético da classe de agregados de 1 a $2 \mathrm{~mm}$, conforme o método do DMP. A linha contínua ilustra como, pel o método proposto, foi obtido o valor do diâmetro médio da mesma classe com auxílio da equação (3) ajustada aos pares de dados, bem como a média das frações de massa acumuladas retidas nessa classe e na classe inferior. No exemplo da figura, o diâmetro médio aritmético da classe foi de 1,50 mm, enquanto o diâmetro médio ajustado foi de $1,37 \mathrm{~mm}$. Com os diâmetros ajustados para todas as classes, utilizou-se a seguinte equação, para calcular o diâmetro médio ajustado (DMA, mm):

$$
D M A=\sum_{i=1}^{n} \frac{m_{i}}{m} \cdot \hat{D}_{i}
$$

em que $\hat{D}_{i}(\mathrm{~mm})$ éo diâmetro médio da classei, obtido segundo o procedimento proposto. O quadro 1 mostra a planilha de cálculo para atingir os valores do DMP e do DMA, utilizando os mesmos dados da figura 1.

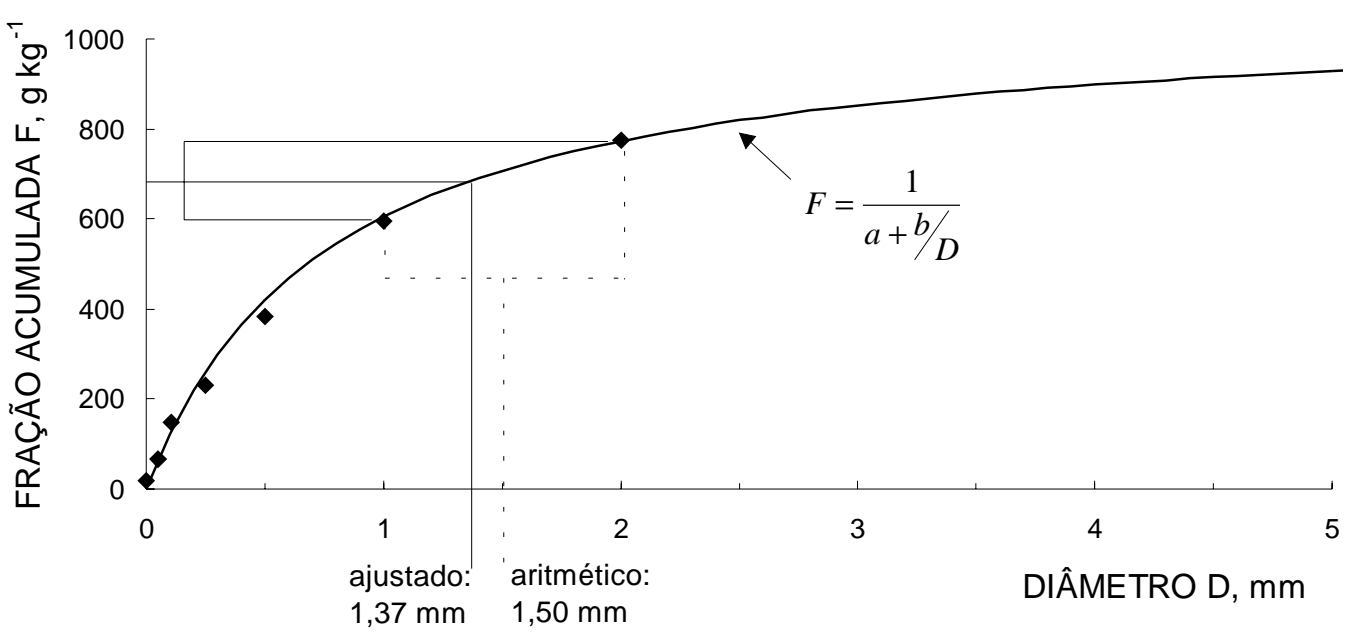

Figura 1. Determinação do diâmetro médio da classe de agregados com diâmetro entre 1 e $2 \mathrm{~mm}$ pelos métodos do DMP (linhas pontilhadas) e do DMA (linhas contínuas), com base em dados obtidos por Carpenedo (1985), num latossolo roxo distrófico sob campo nativo com tratamento úmido, ajustados à equação 3 $(\mathrm{a}=0,933, \mathrm{~b}=0,720)$. 
Quadro 1. Planilha de cálculo do DMP e do DMA

\begin{tabular}{|c|c|c|c|c|c|c|c|c|c|c|}
\hline $\mathbf{i}$ & $D_{i}$ & $\frac{m_{i}}{m}$ & $\mathbf{F}_{\mathrm{i}}$ & $\left(F_{i}+F_{i+1}\right) / 2$ & $\overline{D_{i}}$ & $\hat{D}_{i}$ & $\frac{m_{i}}{m} \overline{D_{i}}$ & & $\frac{m_{i}}{m} \hat{D}_{i}$ & \\
\hline & & $\ldots$ & $\mathrm{kg} \mathrm{kg}^{-1}$ & - & $\ldots$ & $\ldots$ & $\mathrm{mm}$ & 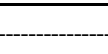 & - & \\
\hline 1 & 9,510 & 0,225 & 1,000 & 0,888 & 5,755 & 3,720 & 1,293 & & 0,836 & \\
\hline 2 & 2,000 & 0,179 & 0,775 & 0,689 & 1,500 & 1,370 & 0,269 & & 0,246 & \\
\hline 3 & 1,000 & 0,213 & 0,596 & 0,490 & 0,750 & 0,648 & 0,160 & & 0,138 & \\
\hline 4 & 0,500 & 0,153 & 0,383 & 0,307 & 0,375 & 0,309 & 0,057 & & 0,047 & \\
\hline 5 & 0,250 & 0,083 & 0,230 & 0,189 & 0,178 & 0,164 & 0,015 & & 0,014 & \\
\hline 6 & 0,105 & 0,082 & 0,147 & 0,106 & 0,078 & 0,085 & 0,006 & & 0,007 & \\
\hline 7 & 0,050 & 0,048 & 0,065 & 0,041 & 0,026 & 0,031 & 0,001 & & 0,001 & \\
\hline \multirow[t]{2}{*}{8} & 0,002 & 0,017 & 0,017 & 0,009 & 0,001 & 0,006 & 0,000 & & 0,000 & \\
\hline & & & & & & & 1,802 & $=\mathrm{DMP}$ & 1,289 & $=\mathrm{DMA}$ \\
\hline
\end{tabular}

$\mathrm{i}=$ número da classe; $\mathrm{D}_{\mathrm{i}}=$ diâmetro superior da classe; $\mathrm{m}_{\mathrm{i}} / \mathrm{m}=$ fração da massa retida na classe; $\mathrm{F}_{\mathrm{i}}=$ fração da massa acumulada, $\overline{D_{i}}=$ diâmetro médio aritmético da classe, $\hat{D}_{i}=$ diâmetro médio da classe (método proposto).

Conforme o pressuposto, a distribuição dos agregados dentro de cada classe é semel hante à distribuição em toda a amostra. Desse modo, em amostras com maior concentração de agregados em classes de maior diâmetro, o DMA será maior que o DMP, no caso contrário, oDMA será menor queoDMP.

\section{EXEMPLO DE APLICAÇÃO}

Os val ores do DMA, cal culados por meio do método proposto, foram comparados com os do DMP e DMG para amostras de diferentes solos, sob diferentes sistemas de preparo e rotação de culturas. Entre as equações (3), (4) e (5), procurou-se sempre utilizar a que apresentava o mai or coeficiente de determinação. Observou-se que, nas equações com $r^{2}$ inferiores a 0,99 , al gumas vezes o val or calculado do DMA não foi confiável, decorrente de grandes desvios entre os pontos observados e a equação ajustada.

Carpenedo (1985) trabal hou com solo col etado da camada de 0-0,10 m de um latossolo roxo distrófico sob campo nativo, preparo convencional e plantio direto, utilizando classes com diâmetros limítrofes de $9,51,2,0,1,0,0,5,0,25,0,105,0,05$ e 0,002 mm, anal isadas por meio do método descrito em Carpenedo \& Mielniczuk (1990). Esses dados demonstraram um melhor ajusteà equação (3), com val ores de $r^{2}$ maiores que 0,98 . Nessas amostras, predominaram agregados menores, resultando em valores do DMA sempre inferiores aos do DMP. Os valores obtidos para oDMG foram ainda menores, em virtude do uso do logaritmo no procedimento de cálculo. Esse procedimento dá maior peso a agregados pequenos, sendo, portanto, adequado para solos com uma predominância de agregados menores, o que explica a ampla utilização do DMG em estudos norte-americanos, cujos solos normal mente apresentam essetipo de distribuição de tamanho de agregados.

Silva (1993) trabalhou com as camadas de 0-0,05 m de um podzólico vermelho-escuro distrófico sob sistemas de culturas aveia-milho e pangola e de um latossol o roxo distrófico sob sistemas de cultura trigosoja e setária. Apesar de os valores dos diâmetros médi os serem maiores que os obtidos com os dados de Carpenedo (1985), a tendência entre DMP, DMG e DMA foi semel hante (Quadro 3).

Quadro 2. Valores obti dos de DMP (equação 1), DMG (equação 2) e DMA (equação 9), com base em dados originais de Carpenedo (1985), após tratamento úmido e seco de material de um latossolo roxo distrófico sob campo nativo, plantio convencional e plantio di reto, bem como o número da equação utilizada na determinação do DMA e os parâmetros a e b e indicadores do ajuste $\mathbf{r}^{2}$ e F

\begin{tabular}{|c|c|c|c|}
\hline & \multicolumn{3}{|c|}{ Sistema de manejo } \\
\hline & \multirow{2}{*}{$\begin{array}{l}\text { Campo } \\
\text { nativo }\end{array}$} & \multicolumn{2}{|c|}{ Plantio } \\
\hline & & Convencional & Direto \\
\hline & \multicolumn{3}{|c|}{ Tratamento úmido } \\
\hline $\mathrm{DMP}(\mathrm{mm})$ & 1,80 & 1,27 & 1,96 \\
\hline DMG $(\mathrm{mm})$ & 0,68 & 0,42 & 0,66 \\
\hline DMA $(\mathrm{mm})$ & 1,29 & 0,95 & 1,27 \\
\hline Equação & (3) & (3) & (3) \\
\hline$a$ & 0,933 & 0,970 & 0,968 \\
\hline$b$ & 0,720 & 0,407 & 0,637 \\
\hline$r^{2}$ & 1,00 & 0,99 & 0,99 \\
\hline \multirow[t]{2}{*}{$\mathrm{F}$} & 1.614 & 595 & 600 \\
\hline & \multicolumn{3}{|c|}{ Tratamento seco } \\
\hline DMP (mm) & 2,31 & 1,60 & 2,11 \\
\hline DMG $(\mathrm{mm})$ & 0,93 & 0,64 & 0,77 \\
\hline DMA $(\mathrm{mm})$ & 1,59 & 1,18 & 1,38 \\
\hline Equação & (3) & (3) & (3) \\
\hline$a$ & 0,899 & 0,928 & 0,955 \\
\hline$b$ & 1,05 & 0,656 & 0,746 \\
\hline$r^{2}$ & 1,00 & 0,99 & 0,99 \\
\hline $\mathrm{F}$ & 3.787 & 526 & 598 \\
\hline
\end{tabular}


Quadro 3. Valores obtidos de DMP (equação 1), DMG (equação 2) e DMA (equação 9), com base em dados originais de Silva (1993), após tratamento úmi do e seco de material de um $P E d$ sob sistemas de culturas aveia-milho e pangola e de um LRd sob sistemas de cultura trigo-soja e setária, bem como o número da equação utilizada na determinação do DMA e os parâmetros a e b e indicadores do ajuste $r^{2}$ e $F$

\begin{tabular}{|c|c|c|c|c|}
\hline & \multicolumn{2}{|c|}{ Ped } & \multicolumn{2}{|c|}{ LRd } \\
\hline & Aveia-milho & Pangola & Trigo-soja & Setária \\
\hline & \multicolumn{4}{|c|}{ Tratamento úmido } \\
\hline $\begin{array}{l}\mathrm{DMP}, \\
\mathrm{mm}\end{array}$ & 1,29 & 2,79 & 1,54 & 3,43 \\
\hline $\begin{array}{l}\text { DMG, } \\
\mathrm{mm}\end{array}$ & 0,48 & 1,08 & 0,65 & 1,84 \\
\hline $\begin{array}{l}\mathrm{DMA} \\
\mathrm{mm}\end{array}$ & 0,88 & 2,26 & 1,10 & 2,35 \\
\hline E quação & (3) & (4) & (3) & (3) \\
\hline a & 0,954 & 0,385 & 0,917 & 0,780 \\
\hline$b_{2}$ & 0,425 & 1,89 & 0,640 & 2,22 \\
\hline$r^{2}$ & 0,99 & 1,00 & 0,99 & 0,99 \\
\hline \multirow[t]{2}{*}{$\mathrm{F}$} & 502 & 1.078 & 708 & 347 \\
\hline & \multicolumn{4}{|c|}{ Tratamento seco } \\
\hline $\begin{array}{l}\text { DMP, } \\
\mathrm{mm}\end{array}$ & 3,93 & 3,95 & 3,29 & 4,13 \\
\hline $\begin{array}{l}\mathrm{DMG}, \\
\mathrm{mm}\end{array}$ & 2,72 & 2,80 & 2,03 & 3,08 \\
\hline $\begin{array}{l}\text { DMA, } \\
\mathrm{mm}\end{array}$ & 3,23 & 3,29 & 2,47 & 3,55 \\
\hline E quação & (3) & (3) & (3) & (3) \\
\hline$a$ & 0,569 & 0,555 & 0,746 & 0,476 \\
\hline$b_{2}$ & 4,10 & 4,23 & 2,42 & 4,98 \\
\hline$r^{2}$ & 1,00 & 1,00 & 1,00 & 1,00 \\
\hline $\mathrm{F}$ & 2.487 & 1.785 & 965 & 1.160 \\
\hline
\end{tabular}

Para avaliar a adequação do método em solos com distintas mineralogias, teores de carbono e granulometria, coletaram-se amostras de solo da camada de 0-0,20 m de sete solos: dois Iatossolos vermelho-escuros (LE 1 e LE 2); um latossolo bruno câmbico ( $\mathrm{LBC}$ ); um brunizém vértico (Bv); um planossolo (PL); uma terra roxa estruturada (TR) e um vertissolo (V). Nesses solos aplicaram-se dois métodos para determinar a estabilidade dos agregados: o método de Carpenedo (1985) e o de Kemper \& Chepil (1965), cujos resultados encontramse no quadro 4.

No método proposto por Carpenedo(1985), verificase que os valores do DMP, DMA e DMG foram inferiores aos obtidos pelo método proposto por Kemper \& Chepil (1965). A diferença entre os métodos está na quantidade de energia que incide sobre os agregados, bem superior no método de Carpenedo (1985). No método de Kemper \& Chepil (1965), utilizaseapenas a fração de agregados entre 4,76 e 9,51 mm, enquanto, no de Carpenedo (1985), utiliza-se toda a amostra (0 a 9,51 mm).

Da mesma maneira que observado nos dados de Carpenedo (1985 ) e Silva (1993), os valores do DMA estão entreos outros dois índices, porém bem próximos do DMP. I sso demonstra que o ajuste das equações resultou em relações parecidas com polinomiais de primeiro grau.

Dos sete sol os avaliados pelo método de Kemper \& Chepil (1965), apenas o planossolo apresentou o coeficiente de determinação inferior a 0,99 (Quadro 4). Nesse caso, aconsel ha-se observar o gráfico com os pontos e a equação ajustada para verificar se existem

Quadro 4. Valores obtidos de DMP (equação 1), DMG (equação 2) e DMA (equação 9), com base em dados originais de Albuquerque (1997), após tratamento úmido - conforme métodos de Carpenedo (1985) e Kemper (1965) - de material de solos do Estado do Rio Grande do Sul, bem como o número da equação utilizada na determinação do DMA e os parâmetros a e b e indicadores do ajuste $\mathbf{r}^{2}$ e F

\begin{tabular}{|c|c|c|c|c|c|c|c|}
\hline & LE 1 & LB & Bv & $\mathbf{P L}$ & TR & $\mathbf{v}$ & LE 2 \\
\hline & \multicolumn{7}{|c|}{ Método de Carpenedo (1985) } \\
\hline $\mathrm{DMP}, \mathrm{mm}$ & 3,34 & 3,07 & 1,88 & 0,96 & 2,47 & 3,37 & 2,11 \\
\hline $\mathrm{DMG}, \mathrm{mm}$ & 1,58 & 1,45 & 0,60 & 0,21 & 1,03 & 1,55 & 0,88 \\
\hline $\mathrm{DMA}, \mathrm{mm}$ & 3,25 & 3,00 & 1,88 & 0,90 & 2,36 & 3,30 & 1,81 \\
\hline Equação & (5) & (5) & (5) & (4) & (4) & (5) & (3) \\
\hline a & 0,303 & 0,336 & 0,532 & 0,841 & 0,376 & 0,293 & 0,931 \\
\hline b & 0,531 & 0,491 & 0,299 & 0,477 & 1,90 & 0,547 & 0,971 \\
\hline$r^{2}$ & 1,00 & 0,98 & 0,98 & 0,99 & 0,99 & 0,99 & 0,99 \\
\hline \multirow[t]{2}{*}{$\mathrm{F}$} & 2.280 & 259 & 243 & 365 & 411 & 590 & 578 \\
\hline & \multicolumn{7}{|c|}{ Método de Kemper (1965) } \\
\hline $\mathrm{DMP}, \mathrm{mm}$ & 6,91 & 7,04 & 5,87 & 2,82 & 5,94 & 6,49 & 6,49 \\
\hline $\mathrm{DMG}, \mathrm{mm}$ & 6,54 & 6,95 & 4,62 & 1,24 & 4,83 & 5,94 & 5,66 \\
\hline $\mathrm{DMA}, \mathrm{mm}$ & 8,61 & 9,21 & 6,30 & 2,44 & 6,55 & 7,78 & 7,62 \\
\hline Equação & (4) & (4) & (4) & (4) & (4) & (4) & (4) \\
\hline a & $-28,2$ & $-114,7$ & $-4,54$ & 0,442 & $-5,41$ & $-14,4$ & $-11,1$ \\
\hline b & 90,0 & 356,7 & 17,1 & 1,80 & 19,8 & 47,4 & 37,4 \\
\hline$r^{2}$ & 1,00 & 1,00 & 0,99 & 0,96 & 1,00 & 1,00 & 1,00 \\
\hline $\mathrm{F}$ & 2.523 & $>9.999$ & 505 & 104 & 954 & $>9.999$ & 1.327 \\
\hline
\end{tabular}


desvios grandes entre os pontos observados e a equação ajustada. Caso existam, recomenda-se encontrar uma equação com coeficiente de determinação superior.

Pel o método de Kemper \& Chepil (1965), os valores de DMA obtidos foram superiores aos do DMP. Por ser menor a energia fornecida aos agregados, bem como a amostra inicial ser composta por agregados grandes relativamenteestáveis, houve predominância de agregados nas peneiras maiores.

Com a finalidade de comparar os valores do DMP, DMG e DMA, fez-se uma regressão linear entre os valores obtidos para esses parâmetros nos exemplos supracitados. A figura 2 mostra os pontos observados com as respectivas retas ajustadas. As regressões resultaram nas seguintes relações entre os parâmetros:

$$
\begin{aligned}
& \text { DMP }=0,887 D M G+1,402\left(r^{2}=0,966\right) \\
& D M P=0,722 \text { DMA +0,995 }\left(r^{2}=0,960\right) \\
& \text { DMG }=0,813 D M A-0,459\left(r^{2}=0,971\right)
\end{aligned}
$$

Pelo teste-t, verificou-se que, ao nível de significância de $95 \%$, os coeficientes angulares das equações (10), (11) e (12) diferem de 1, enquanto os coeficientes lineares diferem de 0 , indicando que há diferença estatística entre os três parâmetros.

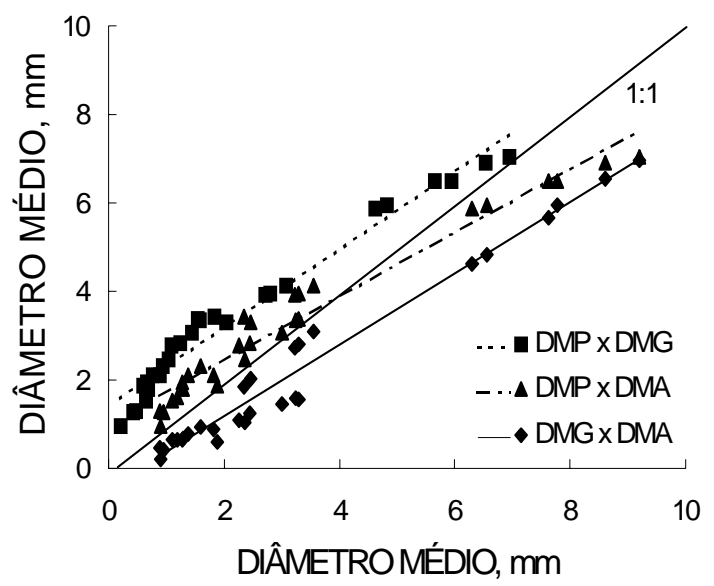

Figura 2. Comparação entre valores do DMP, DMG e DMA com linhas de ajuste (equações 10, 11 e 12).

\section{CONCLUSÕES}

J ustifica-se a utilização do parâmetro DMA no lugar do DMP ou DMG, por ser considerado, no seu cálculo, um diâmetro médio para cada fração que leva em consideração a provável distribuição dos tamanhos dos agregados dentro da fração. Porém, o coeficiente de determinação do ajuste da equação aos dados de massa acumulada e diâmetro superior da classe deve ser alta $(\geq 0,99)$ para o método proposto poder ser aplicado sem ressalvas.

\section{CONSIDE RAÇÃO FINAL}

Encontram-se, à disposição dos interessados, uma planilha (formato MicroSoft Excel 5.0 ou 7.0), que executa o cálculo do DMP, DMG e DMA a partir dos valores de massa retida em cada classe, e os parâmetros de ajuste de uma das equações (3), (4) ou (5), que podem ser obtidos por meio de um programa de regressão não linear (como, por exemplo, o programa TableCurve ${ }^{\mathrm{Tm}}$, da J andel Scientific).

\section{LITE RATURA CITADA}

ALBUQUERQUE, J.A. Efeito de atributos físicos, químicos e mineralógicos na erosão em entressulcos. Porto Alegre, UFRGS, 1997. (Tese de Doutorado - no prelo)

AMEZKETA, E.; SINGER, M.J . \& BISSONAIS, Y. Testing a new procedure for measuring water-stable aggregation. Soil Sci. Soc. Am. J ., Madison, 60:888-894, 1996.

CAMPOS, B.C.; REINERT, D.J .; NICOLODI, R.; RUEDELL, J . \& PETRERE, C. Estabilidade estrutural de um latossolo vermelho-escuro distrófico após sete anos de rotação de culturas e sistemas de manejo do solo. R. Bras. Ci. Solo, Campinas,19:121-126, 1995.

CARPENEDO, V. Qualidade e estabilidade de agregados de latossolo roxo submetido a diferentes manejos. Porto Alegre, UFRGS, 1985. 129p. (Dissertação de Mestrado)

CARPENEDO, V. \& MIELNICZUK, J. Estado de agregação e qualidade de agregados de latossolos roxos submetidos a diferentes sistemas de manejo. R. Bras. Ci. Solo, Campinas, 14:99-105, 1990.

ELLSWORTH, T.R.; CLAPP, C.E. \& BLAKE, G.R. Temporal variations in soil structural properties under corn and soybean cropping. Soil Sci., Baltimore, 151:405-416, 1991.

ELTZ, F.L.F.; PEIXOTO, R.T.G. \& J ASTER, F. Efeitos de sistemas de preparo do sol o nas propriedades físicas e químicas de um latossolo bruno álico. R. Bras. Ci. Solo, Campinas, 13:259267, 1989.

GARDNER,W.R. Representation of soil aggregate-size distribution by a logarithmic-normal distribution. Soil Sci. Soc. Am. Proc., Madison, 20:150-153, 1956.

KEMPER, W.D. \& CHEPIL, W.S. Size distribution of aggregates. In: BLACK, C.A.; EVANS, D.D.; WHITE, J .L.; ENSMINGER, L.E. \& CLARCK, F.E., eds. Methods of soil analysis. Part I. Madison, American Society of Agronomy, 1965. p.499-510. (Agronomy Series, 9)

LAL, R.; VLEESCHAUWER, D. \& NGANJ E, R.M. Changes in properties of a newly cleared tropical alfisol as affected by mulching. Soil Sci. Soc. Am. J., Madison, 44:827-833, 1980.

SHALLER, F.W. \& STOCKINGER, K.R. A comparison of five methods for expressing aggregation data. Soil Sci. Soc. Am. Proc., Madison, 17:310-313, 1953.

SILVA, I.F. Formação, estabilidade e qualidade de agregados do solo afetados pelo uso agrícola. Porto Alegre, UFRGS, 1993. 126p. (Tese de Doutorado) 
STIRK, G.B. Expression of soil aggregate distributions. Soil Sci., Baltimore, 86:133-135, 1958.

UNGER, P.W. Overwinter changes in physical properties of notillage soil. Soil Sci. Soc. Am. J., Madison, 55:778-782, 1991.

VAN BAVEL, C.H.M. Mean weight diameter of soil aggregates as a statistical index of aggregation. Soil Sci. Soc. Am. Proc., Madison, 14:20-23, 1949.
VAN BAVEL, C.H.M. Report of the Committee on Physical Analyses 1951-1953, Soil Science Society of Ameruca. Soil Sci. Soc. Am. Proc., Madison, 17:416-418, 1953.

YODER , R.E. A direct method of aggregate analysis of soils and a study of the physical nature of erosion losses. J. Am. Soc. Agron., Madison, 28:337-351, 1936.

YOUKER, R.E. \& McGUINNESS, J.L. A short method of obtaining mean weight-diameter values of aggregate analyses of soils. Soil Sci., Baltimore, 83:291-294, 1957. 